\title{
Métodos Histoquímicos e Inmunohistoquímicos para la Estimación del Intervalo Postmortem en Tejidos Humanos: Una Revisión
}

\author{
Histochemical and Immunohistochemical Methods for the Postmortem \\ Interval Estimation in Human Tissues: A Review
}

Clivia Guerrero-Urbina ${ }^{1,2,3,4}$; Mariano del Sol ${ }^{1,5} \&$ Gabriel M. Fonseca ${ }^{1,6}$

\begin{abstract}
GUERRERO-URBINA, C.; DEL SOL, M. \& FONSECA, G. M. Métodos histoquímicos e inmunohistoquímicos para la estimación del intervalo postmortem en tejidos humanos: Una revisión. Int. J. Morphol., 38(2):241-246, 2020.

RESUMEN: El intervalo postmortem (IPM) es un importante desafío a resolver en patología forense, y consiste en poder determinar el tiempo transcurrido desde la muerte hasta el momento de la autopsia. Dada la poca confiablidad de algunos métodos por la gran influencia de factores externos, la Histoquímica (HQ) y la Inmunohistoquímica (IHQ), entre otros, han recibido considerable atención por sus niveles de objetividad en la investigación forense. Se presenta una revisión con búsqueda sistemática de estudios experimentales que apliquen métodos HQs e IHQs para la estimación del IPM sobre material cadavérico humano. Se identificaron 1053 artículos de los cuales 12 cumplieron con los criterios, a los que se agregaron 4 mediante una búsqueda manual (n=16 artículos). Alemania fue el país con más publicaciones destacando con 8 artículos. Las técnicas HQs deAgNORs, TRAP y PAS fueron utilizadas en 5 estudios (glándulas sudoríparas, piel, hígado, médula ósea y mucosa labial), mientras que las IHQs fueron empleadas con diferentes grupos antigénicos en 12 estudios (páncreas, cerebro, pulmón, tiroides, hígado, glándulas pituitarias, glándulas sudoríparas y mucosa gingival). Las estimaciones del IPM fueron posibles con márgenes entre 2-3 h. hasta los 20 días dependiendo de la técnica. El análisis de tejidos provenientes de cavidad oral asegura una vía no invasiva, de fácil acceso y bajo resguardo natural a la influencia de factores ambientales. Si bien no existe un método único que permita de manera confiable estas estimaciones, la introducción de nuevas técnicas permitiría evitar la producción de errores.
\end{abstract}

PALABRAS CLAVE: Inmunohistoquímica; Histoquímica; Estimación del intervalo postmortem; Autopsia medicolegal.

\section{INTRODUCCIÓN}

El intervalo postmortem (IPM), o tiempo transcurrido desde la muerte a la autopsia, es uno de los mayores desafíos a resolver de la medicina forense en todo el mundo (Madea et al., 2019). La estimación precisa del IPM permite al médico legista responder a familiares, policía y fiscalía no solo acerca del tiempo transcurrido de la muerte, sino además a esclarecer en muchos casos el contexto en que esa se produjo. El diagnóstico del IPM consiste en identificar los cambios físico-químicos que se presentan en el cadáver, y las técnicas tradicionales utilizadas son empíricas, basadas principalmente en cambios predecibles que ocurren dentro del cuerpo después de la muerte (Mazzotti et al., 2019). Estos cambios se refieren a la temperatura, los producidos en las glándulas sudoríparas, la estimulación química de la musculatura pupilar, la mecánica y eléctrica de la estimulación muscular, así como la extensión del rigor mortis y la lividez postmortem, todos útiles en diferentes periodos de tiempo (Madea et al.; Wehner et al., 2001a).

Para estimar con mayor certeza el IPM, y superando a los estándares tradicionales, se han propuesto diferentes métodos, muchos de los cuales han sido caracterizados como poco confiables debido a innumerables factores modificantes externos. La literatura reporta avances en su valor científico, con la utilización de variadas propuestas desde la química y física para estimar el IPM y con ello, aumentar la certeza de estas estimaciones (Madea et al.).

Se ha mencionado que el estudio de la morfología y la función, cubre no solo todos los campos de las ciencias

\footnotetext{
${ }^{1}$ Programa de Doctorado en Ciencias Morfológicas, Facultad de Medicina, Universidad de La Frontera, Temuco.

${ }^{2}$ Carrera de Obstetricia, Facultad de Ciencias Médicas, Universidad Central del Ecuador, Ecuador.

${ }^{3}$ Unidad de Medicina Legal, Zona 9. Policía Nacional del Ecuador, Ecuador.

${ }^{4}$ Servicio Nacional de Medicina Legal y Ciencias Forenses, Quito, Ecuador.

${ }^{5}$ Centro de Excelencia en Estudios Morfológicos y Quirúrgicos, Universidad de La Frontera, Chile.

${ }^{6}$ Centro de Investigación en Odontología Legal y Forense (CIO), Facultad de Odontología, Universidad de La Frontera, Temuco, Chile.
} 
médicas básicas, sino también los de las ciencias clínicas como la medicina y la cirugía. Sin embargo, este estudio se ha visto claramente potenciado por la Histoquímica (HQ) y la Citoquímica (CQ) (términos que aplican a las reacciones según sus niveles tisular o celular respectivamente) (Nagata, 2008), campos destinados a localizar componentes químicos de células y tejidos en secciones histológicas mediante el uso de diferentes técnicas, analizando funciones y actividad química con base en la morfología (Nagata). Las técnicas HQs, son metodologías histológicas fundamentadas en modificaciones moleculares y en los productos celulares resultantes de las reacciones químicas entre la muestra de tejido biológico y los agentes químicos empleados en dichas técnicas. A diferencia de las coloraciones rutinarias, localizan y reconocen los efectos resultantes de las interacciones mencionadas, además de identificar los productos químicos propios de los tejidos. De esta manera, son de gran utilidad en estudios clínicos e investigaciones científicas partiendo de las funciones y características determinantes de los productos (Charan Gowda et al., 2016). Aunque estas técnicas tuvieron su auge a mediados del siglo pasado, la vigencia de su empleo no se ha visto mermada y su aplicación en medicina y biología es de constante citación en la literatura actual, con sugerencias de utilización incluso en el área forense (Nagata; Zhou, 2017)

Con el avance significativo de las metodologías y recursos diagnósticos, la exposición de simples reacciones químicas derivó a procesos más complejos, permitiendo la visualización incluso de reacciones. Desde aquí, la Inmunohistoquímica (IHQ) ha posibilitado detectar, amplificar y hacer visible un antígeno específico utilizando sistemas de anticuerpos, logrando estudiar y analizar procesos y reacciones con altos niveles de especificidad inmunológica (Nagata). Estas potencialidades han propuesto a la IHQ como una técnica alternativa también de utilidad diagnóstica forense (Ortmann et al., 2017; Lesnikova et al.; 2018). En lo que refiere a la estimación del IPM, estudios experimentales han demostrado que es posible establecer cambios progresivos vinculados al IPM mediante histología e HQ en el curso de las primeras horas postmortem (Charan Gowda et al.). Algunos autores (Wehner et al., 1999; 2000; 2001a; 2001b; 2009) han postulado la posibilidad de emplear métodos IHQs sobre tejidos cadavéricos para correlacionar las reacciones con el IPM. La premisa es que la estructura terciaria del antígeno sufre cambios postmortem, y al aumentar el IPM disminuye la eficacia de la tinción de desnaturalización de proteínas. La aplicación de métodos de IHQ puede ser útil, incluso sin datos de fondo disponibles (Ortmann et al.).

Tomando en consideración que la HQ y la IHQ ofrecen importantes niveles de objetividad a la resolución de casos judiciales representando estrategias de vanguardia en la investigación forense (Bardale et al., 2012; Lesnikova et $a l$.), se presenta una revisión con búsqueda sistemática de la literatura para pesquisar aplicaciones de este tipo de métodos para la estimación del IPM y los potenciales del empleo de estas técnicas en determinados compartimentos orgánicos.

\section{MATERIAL Y MÉTODO}

Se realizó una revisión con búsqueda sistemática de la literatura científica en la base PubMed/MEDLINE a cargo de dos investigadores independientes utilizando la estrategia ("immunohistochemical" OR "histochemistry" OR "cytochemistry") AND "time" AND “death" NOT "cancer" NOT “inflammation”, sin límite temporal. Esta se realizó el 10 de octubre de 2019, incluyendo artículos primarios completos en inglés, español o portugués, centrándose en estudios experimentales que describieran métodos HQs o IHQs para la estimación del IPM sobre material cadavérico humano. Fueron excluidas revisiones bibliográficas, reportes de casos, cartas al editor y estudios que no respondieran al objeto de la investigación. Dentro de las categorías de análisis se consideraron el año y perfil de los autores, protocolo de HQ/IHQ utilizado, tejido humano estudiado y outcomes. Los artículos seleccionados fueron evaluados en su calidad por dos investigadores de manera independiente siguiendo la guía de evaluación crítica de la JAMA, resolviéndose por consenso aquellos casos donde existieron discrepancias.

\section{RESULTADOS}

Se identificaron 1053 artículos como resultado de la búsqueda, de los cuales 12 cumplieron con los criterios de inclusión y presentaron la calidad metodológica requerida. Una búsqueda manual posterior permitió incluir otras 4 publicaciones que reunían similares criterios (Fig. 1). La totalidad de artículos científicos revisados fue de 16 (Tabla I).

Año de publicación. Se identificaron estudios entre los años 1994 y 2019, 5 de los cuales han sido publicados en los últimos 3 años. Es destacable que 3 de esos 5 artículos han focalizado su investigación en la aplicación de métodos IHQs en tejidos obtenidos de la cavidad oral (Mazzotti et al.; Fais et al., 2018; Charan Gowda et al.).

Países y perfiles de los autores. Alemania destacó con 8 artículos (53,33\% del total de documentos), uno de ellos en colaboración con Estados Unidos y Dinamarca (Lesnikova et al.). Cinco de esos artículos fueron firmados por el equi- 


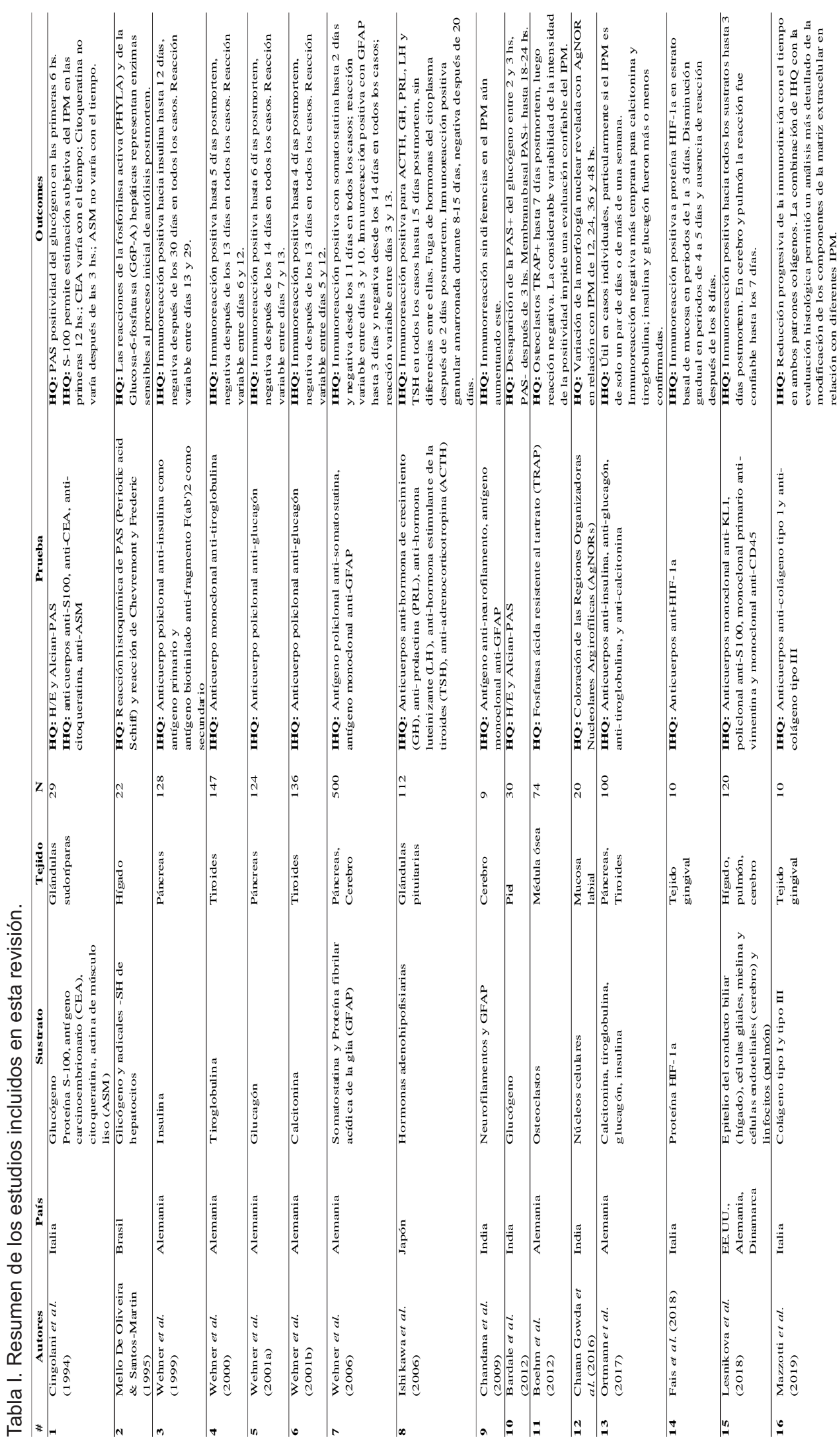

po perteneciente al Instituto de Medicina Forense de la Universidad de Tübingen, liderado por el Dr. Frank Wehner, especialista en Medicina Forense, médico principal en el mencionado instituto desde el año 2001, con su área de investigación focalizada en métodos IHQs en medicina legal (http:// www.laborundmore.com/ research/7623/Dr.-FrankWehner.html).

\section{Italia destacó con 3} artículos, entre los que se identificaron 2 nuevas contribuciones a la aplicación de IHQ para análisis de tejido gingival, por académicos de los Departamentos de Ciencias Médicas y Quirúrgicas (área de Medicina Legal) y de Ciencias Biomédicas y Neuromotoras de la Universidad de Bologna. En el caso de India, también con 3 artículos, se destaca el aporte realizado por Charan Gowda et al., aplicando técnicas HQs en muestras de mucosa labial.

El equipo del Prof. Takaki Ishikawa en el Departamento de Medicina Legal de la Facultad de Medicina de la Universidad de Osaka en Japón, publicó 1 estudio en 2006 aplicando IHQ en glándulas pituitarias. Brasil, si bien también con 1 artículo, destacó junto a Italia por encontrarse entre las primeras contribuciones en HQ a la estimación del IPM (Mello De Oliveira \& SantosMartin, 1995). 


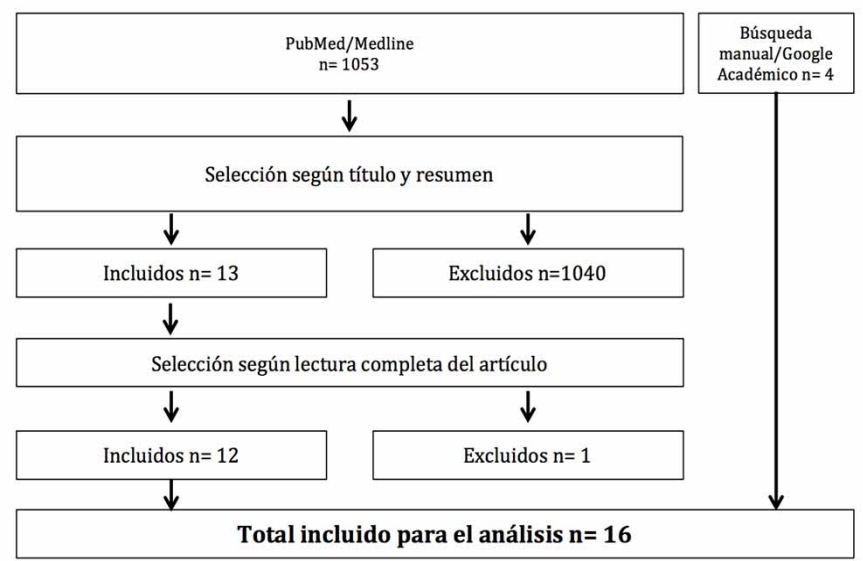

Fig. 1. Flujograma de la revisión de literatura y criterios para selección y exclusión de artículos.

Métodos y muestras analizadas. Cinco estudios emplearon métodos HQs: técnica de coloración de las Regiones Organizadoras Nucleolares Argirofílicas (AgNORs) en mucosa labial (Charan Gowda et al.); técnica de la fosfatasa ácida resistente al tartrato (TRAP) en médula ósea de columna ilíaca (Boehm et al., 2012); y reacción histoquímica de PAS (Periodic acid Schiff) en piel (Bardale et al.), hígado (Mello De Oliveira \& Santos-Martin) y glándulas sudoríparas, en este último caso, también aplicando IHQ sobre las mismas muestras (Cingolani et al., 1994). Sumado el estudio de Cingolani et al., la IHQ fue empleada en 12 estudios con diferentes grupos antigénicos, siendo el páncreas el sustrato de mayor evaluación, con 4 artículos (Ortmann et al.; Wehner et al., 1999; 2001a; 2006), seguido por cerebro y tiroides, ambos con 3 estudios cada uno. Otros sustratos analizados fueron 2 en mucosa gingival (Fais et al.; Mazzotti et al.), y glándulas sudoríparas, glándulas pituitarias, hígado y pulmón, todos con 1 reporte cada uno.

Los mayores tamaños muestrales fueron los analizados por el equipo del Dr. Wehner, con muestras de páncreas y cerebro ( $n=500$ en 2006), Tiroides ( $n=147$ y n=136 en 2000 y 2001b respectivamente) y páncreas ( $\mathrm{n}=128$ y $\mathrm{n}=124$ en 1999 y 2001 respectivamente). La menor estudiada fue la de 9 muestras de cerebro, en India (Chandana et al., 2009). Respecto a las de tejidos orales, el equipo italiano procesó con IHQ a 10 muestras de tejido gingival humano en 2018 y 2019, y en India analizaron 20 de mucosa labial mediante HQ.

Outcomes. En lo que refiere a la HQ, el uso del PAS permitió estimaciones subjetivas confiables del IPM solo dentro de las primeras 2 a 3 horas postmortem en todos los tejidos analizados. Mientras AgNOR en mucosa labial permitió asociar significativamente variaciones de morfología nuclear en relación con el IPM, TRAP en osteoclastos de médula ósea demostró excesiva variabilidad lo que no ofreció confiabilidad para estimar el IPM.
Por otra parte, la IHQ refirió estimaciones confiables del IPM hasta 20 días utilizando anticuerpos anti-hormonas pituitarias (GH, PRL, LH, TSH, ACTH), hasta 12 días con anti-insulina en páncreas, 6 días con anti-glucagón en páncreas, 5 días con anti-tiroglobulina y 4 días con anti-calcitonina -ambos en tiroides-, y 3 días con anti-GFAP y 2 días con anti-somatostatina -ambos en páncreas y cerebro. Ortmann et al., comparando respuestas a calcitonina, tiroglobulina, glucagón e insulina, afirman que estas dos últimas fueron las de mayor confiabilidad. Anticuerpos anti-KL1, anti-vimentina, antiCD45 y anti-s100 en hígado, pulmón y cerebro ofrecieron inmunoreacción positiva hasta 3 días postmortem y confiabilidad hasta 7 días en cerebro y pulmón (Lesnikova et al.).

Particularmente en tejido gingival, los anticuerpos anti-HIF-1a ofrecieron confiabilidad para estimar el IPM hasta 3 días postmortem, mientras que los anticuerpos anticolágeno tipo 1 y anti-colágeno tipo 3 demostraron ser útiles en IPM definidos ya que el colágeno posee índices de baja degradación dependiente del tiempo en comparación con otras proteínas funcionales o estructurales (Mazzotti et al.).

\section{DISCUSIÓN}

En 1966, el Profesor y Director del Departamento de Medicina Forense de la Universidad de Turku en Finlandia, Profesor Jyrki Raekallio, afirmaba: “...me temo que una estimación precisa de la hora de la muerte seguirá siendo imposible, también histoquímicamente, en un futuro próximo", esto apoyándose en que existen demasiadas variables influyendo en este cálculo. Sin embargo, en 2019, uno de los mayores referentes en estimación del IPM el Prof. Burkhard Madea, junto a miembros del Instituto de Medicina Forense en el Hospital Universitario Bonn en Alemania, reportaron un caso en el que, mediante IHQ sobre tiroides y páncreas de una mujer de 36 años hallada muerta cercana a una línea de ferrocarril, lograron estimar el IPM por encima de los 6 días, con un límite confianza del $95 \%$ de $\pm 24 \mathrm{~h}$. Los autores afirman que, aunque la estimación del IPM mediante IHQ solo puede realizarse en plazos de tiempo cercanos a la muerte, igualmente puede ser útil en casos individuales. Probablemente este sea el único caso con aplicación de este tipo de técnicas en campo que se haya reportado, con lo que los autores sugieren más estudios de control sobre diferentes condiciones ambientales y de temperatura (Madea et al.). 
Si bien se ha mencionado que los métodos morfológicos para la estimación del IPM no poseen valor práctico en la mesa de autopsia, parece existir un cambio de paradigma en lo que refiere a la aplicación de métodos HQ e IHQ para este fin (Madea et al.): desde las contribuciones de Wehner et al. hasta la presentación de tablas específicas para aplicar en campo (Madea et al.), el progreso de la investigación en esta área ha dado resultados prometedores. Bisker \& RalebitsoSenior (2018) mencionan que, a pesar de la considerable inversión que significan y del escaso valor práctico en casos reales (debido a sus faltas de precisión, consistencia y confiabilidad), algunos enfoques de IHQ pueden poseer aplicabilidad potencial, especialmente cuando se usan en combinación con otras técnicas.

La aplicabilidad de un método para estimar el IPM depende no solo de su confiabilidad sino además de la accesibilidad del tejido a evaluar, de sus costos implícitos y del tiempo de su procesamiento (Madea et al.). Desde ese punto de vista, la aplicación de técnicas diagnósticas histológicas y moleculares para la estimación del IPM parecen representar hoy un papel esencial en la práctica de la patología forense (Mazzotti et al.). Ya el estudio realizado en 1994 por Cingolani et al. sobre glándulas sudoríparas de muestras seriadas de piel tomadas a diferentes IPM, artículo precursor en la temática y con utilización de tres tipos diferentes de pruebas: HQ (H/E y Alcian-PAS), IHQ (S-100, CEA, Citoqueratina y ASM) y ultraestructural (microscopía electrónica), concluyó que, aunque el análisis ultraestructural logró identificar transformaciones específicas para cada IPM, la HQ y la IHQ proporcionaron también información útil no solo para las etapas cronológicas consideradas, sino también para las fases individuales ( 3 h. para H/E y 6 hs. para Alcian-PAS).

Zhou, ha propuesto una redefinición de la HQ: "con base en la histología, son los métodos y tecnologías modernos en física, química, bioquímica, inmunología y biología molecular que se ofrecen para la detección de composiciones químicas in situ, y los análisis cualitativos realizados para comprender las reglas normales y anormales de los metabolismos, funciones y cambios morfológicos en células y tejidos". Sus contenidos comprenden estudios sobre material celular orgánico e inorgánico, diferentes tipos de enzimas, antígenos y anticuerpos en células y tejidos y fragmentos genéticos endógenos y exógenos; siendo una de las ciencias de la vida de más rápido desarrollo tanto en sus métodos de fijación como en sus marcadores celulares, el uso de citometría de flujo y el microscopio confocal de escaneo láser -entre otras nuevas tecnologías-, como en el resguardo y análisis de imágenes experimentales (fotomicrografía), la HQ hoy se presenta como un recurso inestimable en diferentes especialidades entre las que puede considerarse a la medicina forense (Zhou). Así mismo, Cabreri- zo Medina et al. (2015) mencionan que aunque existen numerosos reportes del uso de la HQ y la IHQ en patología forense, estos métodos siguen sin ser contrastados, "entre otras razones por la variabilidad de las circunstancias de la muerte, de la etiología de las lesiones y del tiempo que pasa desde que se produce la muerte hasta que se recogen las muestras y se fijan en formol para su posterior estudio histopatológico". Cingolani et al. van aún más allá al proponer a la HQ y la IHQ como recursos sumamente útiles para poder elaborar diagnósticos objetivos y adecuados.

Lesnikova et al., afirman que la IHQ es utilizada menos frecuentemente por los patólogos forenses que por los patólogos quirúrgicos, bajo los argumentos de que: 1) sus tinciones aumentan los costos y el tiempo de respuesta; 2) la precisión diagnóstica puede no ser una necesidad en casos específicos y; 3) la técnica puede no funcionar bien en muestras postmortem con lo que el rendimiento diagnóstico disminuido no justificaría gastos adicionales. Así mismo, los autores resuelven estas dudas al afirmar que, si bien los costos pueden representar el mayor problema, la IHQ no afecta significativamente el tiempo de respuesta para una autopsia ya que la mayoría de los laboratorios de histopatología pueden realizar las tinciones con anticuerpos comúnmente utilizados, dentro de los primeros dos días.

La evaluación de tejidos provenientes de la cavidad oral para estimación del IPM se focalizó en 3 estudios detectados en esta revisión, aplicando métodos IHQs en mucosa labial (Charan Gowda et al.) y tejido gingival (Fais et al.; Mazzotti et al.). Eso se suma a la tendencia iniciada años antes con propuestas de evaluaciones de cambios histológicos para estimar el IPM en esos mismos tejidos (Pradeep et al., 2009; Yadav et al., 2012; 2015); Mahalakshmi et al., 2016; Muthukrishnan et al., 2018). Yavad et al. (2015), afirman que existe mucha información publicada respecto a los fenómenos postmortem en tejidos orales, lo que podría restringir el rol de los médicos forenses al momento de estimar el IPM. Mazzotti et al. afirman que al igual que otras células del tejido corporal, la mucosa oral pierde su morfología normal como resultado de la autólisis post mortem y estos cambios celulares y subcelulares ya han sido considerados un criterio útil para estimar el IPM. Estos autores coinciden en que la cavidad oral representa una vía no invasiva y de fácil acceso para la obtención de muestras a evaluar, a la vez que supone un resguardo natural a la influencia de factores ambientales responsables de una descomposición más rápida tisular (Yadav et al., 2012; Mazzotti et al.).

Coincimos con Yavad et al. (2015) en que no existe método lo suficientemente confiable como para ser utilizado como único para estimar el IPM, y que la introducción de nuevas técnicas prevendrá la producción de errores diag- 
nósticos. La HQ -más simple, rápida y de bajo costo-, y la IHQ -superando a estas alturas sus etapas experimentales con resultados promisorios y superadores respecto de los meramente morfológicos (Lesnikova et al.)- se ofrecen como herramientas de evolución natural en respuesta a las exigencias médico legales actuales.

GUERRERO-URBINA, C.; DEL SOL, M. \& FONSECA, G. M. Histochemical and immunohistochemical m ethods for the postmortem interval estimation in human tissues: A review. Int. J. Morphol., 38(2):241-246, 2020.

SUMMARY: The postmortem interval (IPM) is an important challenge to be solved in forensic pathology, and it consists in determine the time elapsed since death until the autopsy. Given the low reliability of some methods due to the great influence of external factors, Histochemistry (HQ) and Immunohistochemistry (IHQ), among others, have received considerable attention for their levels of objectivity in forensic investigation. A scoping review of experimental studies that apply HQs and IHQs methods to estimate the MPI on human cadaveric material is presented. We identified 1053 articles, of which 12 met the criteria; we added 4 articles through a manual search ( $n=16$ articles). Germany was the most productive country, with 8 articles. HQ techniques of AgNORs, TRAP and PAS were used in 5 studies (on sweat glands, skin, liver, bone marrow and labial mucosa), while IHQs techniques were used with different antigenic groups in 12 studies (on pancreas, brain, lung, thyroid, liver, pituitary glands, sweat glands and gingival mucosa). IPM estimates were possible with margins between 2-3 hours up to 20 days depending on the technique. The analysis of oral tissues ensures a non-invasive route, easily accessible and under natural protection to the influence of environmental factors. Although there is no single method that reliably allows these estimates, the introduction of new techniques would prevent the production of errors.

KEY WORDS: Immunohistochemistry; histochemistry; Postmortem interval estimation; Medicolegal autopsy.

\section{REFERENCIAS BIBLIOGRÁFICAS}

Bardale, R. V.; Tumram, N. K.; Dixit, P. G. \& Deshmukh, A. Y. Evaluation of histologic changes of the skin in postmortem period. Am. J. Forensic Med. Pathol., 33(4):357-61, 2012.

Bisker, C. \& Ralebitso-Senior, T. K. The Method Debate: A State-of-the-Art Analysis of PMI Estimation Techniques. En: Ralebitso-Senior, T. K. (Ed.). Forensic Ecogenomics: The applicaton of microbial ecology analysis in forensic contexts. London, Academic Press, 2018.

Boehm, J.; Schmidt, U.; Porsche, M.; Veeck, J. \& Schaefer, H. E. Post-mortem analysis of bone marrow osteoclasts using tartrate-resistant acid phosphatase staining: does histochemistry work and correlate with time since death? $J$. Clin. Pathol., 65(11):1013-8, 2012.

Cabrerizo Medina, E.; Villanueva de la torre, H. \& Salguero Villadiego, M. Estudio histopatológico de la evolución temporal de las lesiones. Cuad. Med. Forense, 21(3-4):127-34, 2015.

Chandana, R.; Mythri, R. B.; Mahadevan, A.; Shankar, S. K. \& Srinivas Bharath, M. M. Biochemical analysis of protein stability in human brain collected at different post-mortem intervals. Indian J. Med. Res., 129(2):189-99, 2009.

Charan Gowda, B. K.; Mohan, C. V.; Hemavathi, S. Nuclear Organizer Region in Establishing Postmortem Interval. Medico-Legal Update, 16(1):150-3, 2016.

Cingolani, M.; Osculati, A.; Tombolini, A.; Tagliabracci, A.; Ghimenton, C. \& Ferrara, S. D. Morphology of sweat glands in determining time of death. Int. J. Legal Med., 107(3):132-40, 1994.
Fais, P.; Mazzotti, M. C.; Teti, G.; Boscolo-Berto, R.; Pelotti, S. \& Falconi, M. HIF1a protein and mRNA expression as a new marker for post mortem interval estimation in human gingival tissue. J. Anat., 232(6):1031-7, 2018.

Ishikawa, T.; Zhu, B. L.; Li, D. R.; Zhao, D.; Michiue, T. \& Maeda, H. Postmortem stability of pituitary hormones in the human adenohypophysis. Leg. Med. (Tokyo), 8(1):34-8, 2006.

Lesnikova, I.; Schreckenbach, M. N.; Kristensen, M. P.; Papanikolaou, L. L. \& Hamilton-Dutoit, S. Usability of Immunohistochemistry in Forensic Samples With Varying Decomposition. Am. J. Forensic Med. Pathol., 39(3):185-91, 2018.

Madea, B., Ortmann, J. \& Doberentz, E. Estimation of the time since death-even methods with a low precision may be helpful in forensic casework. Forensic Sci. Int., Jul 27:109879, 2019.

Mahalakshmi, V.; Gururaj, N.; Sathya, R.; Sabarinath, T. R.; Sivapathasundharam, B. \& Kalaiselvan, S. Assessment of histological changes in antemortem gingival tissues fixed at various time intervals: A method of estimation of postmortem interval. J. Forensic Dent. Sci., 8(2): 114, 2016.

Mazzotti, M. C.; Fais, P.; Palazzo, C.; Fersini, F.; Ruggeri, A.; Falconi, M. \& Teti, G. Determining the time of death by morphological and immunohistochemical evaluation of collagen fibers in postmortem gingival tissues. Leg. Med. (Tokyo), 39:1-8, 2019

Mello de Oliveira, J. A. \& Santos-Martin, C. C. Enzyme histochemistry of the liver in autopsy material at different post-mortem times. Med. Sci. Law, 35(3):201$6,1995$.

Muthukrishnan, S.; Narasimhan, M.; Paranthaman, S. K.; Hari, T.; Viswanathan, P. \& Rajan, S. T. Estimation of time since death based on light microscopic, electron microscopic, and electrolyte analysis in the gingival tissue. J. Forensic Dent. Sci., 10(1):34-9, 2018

Nagata, T. Histochemistry, General and Special. Annu. Rev. Biomed. Sci., 10:105$59,2008$.

Ortmann, J.; Doberentz, E. \& Madea, B. Immunohistochemical methods as an aid in estimating the time since death. Forensic Sci. Int., 273:71-9, 2017.

Pradeep, G. L.; Uma, K.; Sharada P. \& Prakash, N. Histological assessment of cellular changes in gingival epithelium in ante-mortem and post-mortem specimens. J. Forensic Dent. Sci., 1(2):61-5, 2009.

Raekallio, J. Applications of histochemistry to forensic medicine. Med. Sci. Law, 6(3): 142-6, 1966

Wehner, F.; Steinriede, A.; Martin, D. \& Wehner, H. D. Two-tailed delimitation of the time of death by immunohistochemical detection of somatostatin and GFAP. Forensic Sci. Med. Pathol., 2(4):241-7, 2006.

Wehner, F.; Wehner, H. D.; Schieffer, M. C. \& Subke, J. Delimitation of the time of death by immunohistochemical detection of insulin in pancreatic beta-cells. Forensic Sci. Int., 105(3):161-9, 1999.

Wehner, F.; Wehner, H. D.; Schieffer, M. C. \& Subke, J. Delimitation of the time of death by immunohistochemical detection of thyroglobulin. Forensic Sci. Int., 110(3): 199-206, 2000

Wehner, F.; Wehner, H. D. \& Subke, J. Delimitation of the time of death by immunohistochemical detection of glucagon in pancreatic a-cells. Forensic Sci. Int., 124(2-3):192-9, 2001a.

Wehner, F.; Wehner, H. D. \& Subke, J. Delimitation of the time of death by immunohistochemical detection of calcitonin. Forensic Sci. Int., 122(2-3):8994, 2001b

Yadav, A.; Angadi, P. V.; Hallikerimath, S.; Kale, A. \& Shetty, A. Applicability of histologic post-mortem changes of labial mucosa in estimation of time of death - a preliminary study. Aust. J. Forensic Sci., 44(4):343-52, 2012.

Yadav, A. B.; Angadi, P. V.; Kale, A. D. \& Yadav, S. K. Histological assessment of cellular changes in postmortem gingival specimens for estimation of time since death. J. Forensic Odontostomatol., 33(1):19-26, 2015.

Zhou, J. Histochemistry. Berlín, Gruyter, 2017.

\section{Dirección para correspondencia:}

Dr. Gabriel M. Fonseca

Centro de Investigación en Odontología Legal y Forense $(\mathrm{CIO})$

Facultad de Odontología

Universidad de La Frontera

Francisco Salazar 01145

Temuco - CHILE

Recibido : 09-09-2019

Aceptado: 14-11-2019

E-mail: gabriel.fonseca@ufrontera.cl 